\title{
Erratum
}

\section{The VLT-FLAMES survey of massive stars: observations centered on the Magellanic Cloud clusters NGC 330, NGC 346, NGC 2004, and the N11 region}

\author{
C. J. Evans ${ }^{1}$, D. J. Lennon ${ }^{2}$, S. J. Smartt ${ }^{3}$, and C. Trundle ${ }^{3}$ \\ 1 UK Astronomy Technology Centre, Royal Observatory Edinburgh, Blackford Hill, Edinburgh, EH9 3HJ, UK \\ e-mail: cje@roe.ac.uk \\ 2 The Isaac Newton Group of Telescopes, Apartado de Correos 321, 38700 Santa Cruz de La Palma, Canary Islands, Spain \\ 3 Department of Physics \& Astronomy, Queen's University Belfast, Belfast BT7 1NN, Northern Ireland, UK
}

A\&A 456, 623-638 (2006), DOI: 10.1051/0004-6361:20064988

Key words. stars: early-type - stars: emission-line, Be - stars: fundamental parameters - Magellanic Clouds binaries: spectroscopic - errata, addenda

Incorrect photometry was given for a total of ten bright stars in Tables 6 and 7 from Evans et al. (2006). These targets were saturated in the Wide Field Imager (WFI) frames and should have been replaced with published values as given below in Table 1. Values for the two stars in N11 are taken from Parker et al. (1992). NGC 2004-003 and NGC 2004-008 are from Balona \& Jerzykiewicz (1993), with the remaining six from Ardeberg et al. (1972). The only consequence of these changes for the published paper is the position of these stars in the Hertzsprung-Russell diagrams in Fig. 12. These were used for a qualitative discussion of the populations in each FLAMES field - because of the difference in reddenings the two apparently massive stars in $\mathrm{N} 11$ (with $\log \left(T_{\text {eff }}\right) \sim 4.2$ and 4.3 ) will have lower luminosities, therefore corresponding to lower-mass evolutionary tracks.

We have also noticed two typographical errors. The classification for N11-020 should be given in Sect. 5.5, 8.2 and Table 6 as O5 I(n)fp. Secondly, in the heading of Table 7, the number in the parentheses after $\alpha$ should, of course, read "(2000)".
Table 1. Replacement photometry for bright stars in Tables 6 and 7 of the published version.

\begin{tabular}{lcccr}
\hline \hline ID & $\alpha(2000)$ & $\delta(2000)$ & $V$ & $B-V$ \\
\hline N11-001 & 045708.85 & -662325.1 & 11.35 & 0.06 \\
N11-002 & 045623.51 & -662951.7 & 11.90 & 0.37 \\
\hline NGC 2004-001 & 053007.07 & -671543.3 & 11.46 & 0.05 \\
NGC 2004-002 & 053112.82 & -671508.0 & 11.60 & 0.09 \\
NGC 2004-003 & 053040.40 & -671609.0 & 12.09 & -0.06 \\
NGC 2004-004 & 053127.90 & -672443.9 & 11.95 & 0.00 \\
NGC 2004-005 & 052942.61 & -672047.5 & 11.93 & 0.04 \\
NGC 2004-006 & 053001.22 & -671436.9 & 12.01 & 0.07 \\
NGC 2004-007 & 053200.76 & -672022.6 & 12.04 & -0.03 \\
NGC 2004-008 & 053040.10 & -671637.9 & 12.43 & -0.03 \\
\hline
\end{tabular}

\section{References}

Ardeberg, A., Brunet, J.-P., Maurice, E., \& Prévot, L. 1972, A\&AS, 6, 249 Balona, L., \& Jerzykiewicz, M. 1993, MNRAS, 260, 782

Evans, C. J., Lennon, D. J., Smartt, S. J., \& Trundle, C. 2006, A\&A, 456, 623 Parker, J. W., Garmany, C. D., Massey, P., \& Walborn, N. R. 1992, AJ, 103, 1205 